\title{
A new ankylosaurid from the late Cretaceous Two Medicine Formation of Montana, USA
}

Paul Penkalski

Acta Palaeontologica Polonica 59 (3), 2014: 617-634 doi: http://dx.doi.org/10.4202/app.2012.0125

Oohkotokia horneri gen. et sp. nov. is described based on a specimen in the collections of the Museum of the Rockies, Montana, USA. Oohkotokia exhibits a unique combination of characters not seen in other late Campanian North American ankylosaurids: prominent, horn-like, trihedral squamosal bosses, a small, undistinguished median nasal plate on the dorsal surface of the rostrum, a relatively small occipital condyle, a smooth, finely pitted osteoderm external texture, and triangular lateral osteoderms. Other specimens from the Two Medicine Formation are referable to Oohkotokia. O. horneri, Euoplocephalus tutus, Dyoplosaurus acutosquameus, and Scolosaurus cutleri separate stratigraphically.

Key words: Dinosauria, Ornithischia, Ankylosauridae, ankylosaur, systematics, Campanian, Cretaceous, Two Medicine Formation, Montana, USA.

Paul Penkalski [pgpenkal@gmail.com], The Geology Museum, 1215 W. Dayton St., Madison, WI. 53706, USA.

This is an open-access article distributed under the terms of the Creative Commons Attribution License (for details please see creativecommons.org), which permits unrestricted use, distribution, and reproduction in any medium, provided the original author and source are credited. 